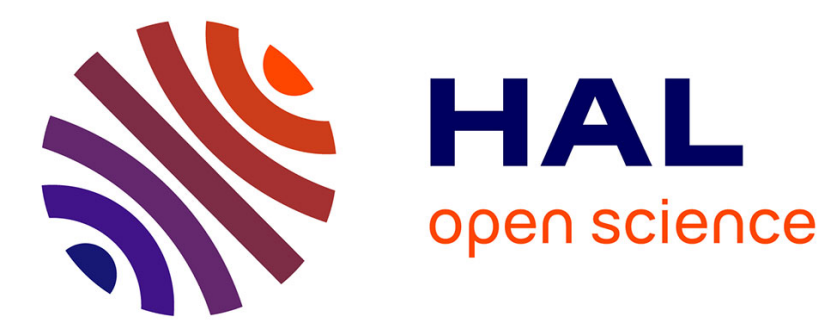

\title{
Type II General Inverse Exponential family of distributions
}

\author{
Farrukh Jamal, Christophe Chesneau, Mohammed Elgarhy
}

\section{To cite this version:}

Farrukh Jamal, Christophe Chesneau, Mohammed Elgarhy. Type II General Inverse Exponential family of distributions. 2018. hal-01914244

\section{HAL Id: hal-01914244 \\ https://hal.science/hal-01914244}

Preprint submitted on 6 Nov 2018

HAL is a multi-disciplinary open access archive for the deposit and dissemination of scientific research documents, whether they are published or not. The documents may come from teaching and research institutions in France or abroad, or from public or private research centers.
L'archive ouverte pluridisciplinaire HAL, est destinée au dépôt et à la diffusion de documents scientifiques de niveau recherche, publiés ou non, émanant des établissements d'enseignement et de recherche français ou étrangers, des laboratoires publics ou privés. 


\title{
Type II General Inverse Exponential family of distributions
}

\author{
Farrukh Jamal $^{a}$, Christophe Chesneau ${ }^{b}$, Mohammed Elgarhy ${ }^{c}$ \\ ${ }^{a}$ Department of Statistics, The Islamia University Bahawalpur, Pakistan. \\ ${ }^{b}$ LMNO, University of Caen, France. \\ ${ }^{c}$ University of Jeddah, Jeddah, Kingdom of Saudi Arabia.
}

\begin{abstract}
In this paper, we introduce a new family of distributions based on the T-X transformation, the inverse exponential distribution, the odds function and the Lehmann type II distribution. We investigate its general mathematical properties, including moments, moment generating function, quantile function, entropies and order statistics. A statistical model is constructed from a special case of the family using the Bur III distribution (also known as exponentiated Lomax distribution) as baseline. The estimation of the parameters are performed by the maximum likelihood method and the least square method. Finally, we illustrate its importance by means of two applications to real life data sets.

Keywords: T-X transformation, Inverse exponential distribution, Maximum likelihood estimation.

2000 MSC: 60E05, 62E15, 62F10.
\end{abstract}

\section{Introduction}

In recent years, numerous methods have been introduced to increase the flexible properties of classic probability distributions. One of the most famous of them is the so called T-X transformation introduced by Alzaatreh et al. (2013). The related T$\mathrm{X}$ family of distributions is characterized by a cumulative distribution function (cdf) described below. Let $(a, b) \in \mathbb{R}^{2}$ with $a<b, p(t)$ is a probability density function (pdf) with support $[a, b], G(x)$ a cdf and $W(x)$ a function such that $W[G(x)]$ satisfies the three following conditions : i) $W[G(x)] \in[a, b]$, ii) $W[G(x)]$ is differentiable and monotonically non-decreasing, iii) $W[G(x)] \rightarrow a$ when $x \rightarrow-\infty$ and $W[G(x)] \rightarrow b$ when $x \rightarrow+\infty$. Then the cdf of the T-X family is given by

$$
F(x)=\int_{a}^{W[G(x)]} p(t) d t, \quad x \in \mathbb{R} .
$$

Among the numerous members of the T-X family, all involving new configurations for $p(t), W(x)$ and $g(x)$, there are the Exp-G (Kw-G type 2) family by Cordeiro et

Email address: ${ }^{a}$ farrukhjamalmphil@gmail.com, ${ }^{b}$ christophe.chesneau@unicaen.fr $\left(\right.$ Farrukh Jamal $^{a}$, Christophe Chesneau ${ }^{b}$, Mohammed Elgarhy $^{c}$ ) 
al. (2013), the Weibull-X family by Alzaatreh et al. (2013), the Gamma-X family by Alzaatreh et al. (2014), the Exponentiated T-X family by Alzaghal et al. (2013), the Weibull-G family by Bourguignon et al. (2014), the Logistic-G family by Torabi and Montazari (2014), The T-Burr family by Nasir et al. (2017), the Ofr family by Haq and Elgarhy (2018) and the TIIGE family by Hamedani et al. (2018). All these families demonstrate nice theoretical and practical properties, offering new solutions in terms of statistical models for the practitioners.

Let us now present and motivate the considered distribution in this paper. Adopting the notations related the cdf of the T-X family given by (1), we consider for $p(t)$ the pdf of the inverse exponential distribution defined by

$$
p(t)=\frac{\lambda}{t^{2}} e^{-\frac{\lambda}{t}}, \quad t \in(0,+\infty)
$$

(observe that it is also a special case of the Frêchet distribution), implying that $a=0$ and $b=+\infty$, and the function $W(x)$ given by

$$
W(x)=\frac{1}{(1-x)^{\theta}}-1 .
$$

By taking any cdf $G(x)$, one can observe that $W[G(x)]$ satisfies the three required conditions, i.e. i), ii) and iii). Note that $W[G(x)]$ can be written as the odds function of a cdf $G_{*}(x)$ belonging to the Lehmann type II distribution with cdf $G(x)$ and parameter $\theta$, i.e. $W[G(x)]=\frac{G_{*}(x)}{1-G_{*}(x)}$, with $G_{*}(x)=1-[1-G(x)]^{\theta}$. Further details on the Lehmann type II distribution can be found in Gupta et al. (1998). Another point of view is to view $W(x)$ as the quantile function of the Lomax distribution with parameters $\alpha=\frac{1}{\theta}$ and $\beta=1$, i.e. with cdf $H(x)=1-(1+x)^{-\frac{1}{\theta}}$. With the presented functions, the cdf $F(x)$ given by (1) becomes

$$
F(x)=\int_{0}^{W[G(x)]} \frac{\lambda}{t^{2}} e^{-\frac{\lambda}{t}} d t=\left[e^{-\frac{\lambda}{t}}\right]_{0}^{W[G(x)]}=e^{-\frac{\lambda}{W[G(x)]}}=e^{-\lambda \frac{[1-G(x)]^{\theta}}{1-[1-G(x)]^{\theta}}} .
$$

For the purpose of this paper, we call the related family of distributions the TIIGIE family (for Type II Generalized Inverse Exponential). The pdf of the general Frêchet distribution for $p(t)$ has ever been considered in Haq and Elgarhy (2018) but with a completely different function $W(x)$. On the other side, the considered function $W(x)$ has ever been considered (as a quantile function) in Nasir et al. (2017) but with a completely different pdf $p(t)$. To the best of our knowledge, the TIIGIE family is new in the literature. This paper deals with a complete mathematical and practical studies of this family, with discussions. We first present the expressions of the crucial functions related to the family, then obtain useful expansions of the pdf, cdf and hrf, a general expression for the quantile function, probability weighted, ordinary and incomplete moments, generating function, entropies and order statistics, with moments. Practical investigations are done for special cases, showing that the TIIGIE family is a quite flexible family of distributions to fit real data from several fields. To be more specific, from Figures 1, 2, and 3 presented in a next section, we can see that the possible pdf shapes 
of TIIGIE family are J shape, reverse J shape, right skewed, left skewed and symmetrical. This means that the TIIGIE family can show suitable fit to those data sets, whose histograms are similar to the TIIGIE family pdfs shapes. Further, the TIIGIE family exhibits monotone [increasing (IFR) and decreasing (DFR)], non-monotone [bathtub (BT) and upside-down bathtub (UBT)] and decreasing-increasing-decreasing (DID) hrf shapes to cope with all types of lifetime data sets. This potentiality is illustrated with applications to two real life data sets, by considering the Bur III distribution as baseline and the maximum likelihood method for the estimations of the parameters.

The rest of the paper is organized as follows. Section 2 studies some immediate properties of the functions characterizing the TIIGIE family. Some special cases with plots are presented in Section 3. A comprehensive account of some of its mathematical properties is proposed in Section 4. Section 5.1 provides the necessary to the estimation of the unknown parameters with the maximum likelihood method and the least square method, with a short simulation study. Applications to two real life data sets are given in Section 6.

\section{On the crucial functions of the TIIGIE family}

\subsection{Some expressions}

We now give the general expressions of the reliability function (rf), the pdf and the hrf of the TIIGIE family. The rf is given by

$$
R(x)=1-F(x)=1-e^{-\lambda \frac{[1-G(x)]^{\theta}}{1-[1-G(x)]^{\theta}}}, \quad x \in \mathbb{R} .
$$

Let us now denote by $g(x)$ the pdf related to $G(x)$. By the derivation of $F(x)$, the pdf is given by

$$
f(x)=\frac{\lambda \theta g(x)[1-G(x)]^{\theta-1}}{\left(1-[1-G(x)]^{\theta}\right)^{2}} e^{-\lambda \frac{[1-G(x)]^{\theta}}{1-[1-G(x)]^{\theta}}}, \quad x \in \mathbb{R} .
$$

The hrf is given by

$$
h(x)=\frac{f(x)}{R(x)}=\frac{\lambda \theta g(x)[1-G(x)]^{\theta-1}}{\left(1-[1-G(x)]^{\theta}\right)^{2}\left[1-e^{-\lambda \frac{[1-G(x)]^{\theta}}{1-[1-G(x)]^{\theta}}}\right]} e^{-\lambda \frac{[1-G(x)]^{\theta}}{1-[1-G(x)]^{\theta}}}, \quad x \in \mathbb{R} .
$$

\subsection{Shapes of the $p d f$ and $h r f$}

The shapes of the pdf and the hrf of the TIIGIE family can be described analytically. As usual, the critical points of the pdf $f(x)$ are the roots of the equation given by $\frac{\partial}{\partial x} \ln (f(x))=0$ with

$$
\frac{\partial}{\partial x} \ln (f(x))=\frac{g^{\prime}(x)}{g(x)}-(\theta-1) \frac{g(x)}{1-G(x)}-2 \theta \frac{[1-G(x)]^{\theta-1} g(x)}{1-[1-G(x)]^{\theta}}+\lambda \theta \frac{[1-G(x)]^{\theta-1} g(x)}{\left(1-[1-G(x)]^{\theta}\right)^{2}} .
$$

This equation can have more than one root. If $x_{*}$ is a root of this equation, then it corresponds to a local maximum if $\frac{\partial^{2}}{\partial x^{2}} \ln \left(f\left(x_{*}\right)\right)<0$, a local minimum if $\frac{\partial^{2}}{\partial x^{2}} \ln \left(f\left(x_{*}\right)\right)>$ 0 and a point of inflection if $\frac{\partial^{2}}{\partial x^{2}} \ln \left(f\left(x_{*}\right)\right)=0$. 
In a similar way, the critical points of the hrf $h(x)$ are the roots of the equation given by $\frac{\partial}{\partial x} \ln (h(x))=0$ with

$$
\begin{aligned}
\frac{\partial}{\partial x} \ln (h(x))= & \frac{g^{\prime}(x)}{g(x)}-(\theta-1) \frac{g(x)}{1-G(x)}-2 \theta \frac{[1-G(x)]^{\theta-1} g(x)}{1-[1-G(x)]^{\theta}}+\lambda \theta \frac{[1-G(x)]^{\theta-1} g(x)}{\left(1-[1-G(x)]^{\theta}\right)^{2}} \\
+ & \frac{\lambda \theta g(x)[1-G(x)]^{\theta-1}}{\left(1-[1-G(x)]^{\theta}\right)^{2}\left[1-e^{-\lambda \frac{[1-G(x)]^{\theta}}{1-[1-G(x)]^{\theta}}}\right]} e^{-\lambda \frac{[1-G(x)]^{\theta}}{1-[1-G(x)]^{\theta}}} .
\end{aligned}
$$

Again, this equation can have more than one root. If $x_{*}$ is a root of this equation, then it corresponds to a local maximum if $\frac{\partial^{2}}{\partial x^{2}} \ln \left(h\left(x_{*}\right)\right)<0$, a local minimum if $\frac{\partial^{2}}{\partial x^{2}} \ln \left(h\left(x_{*}\right)\right)>0$ and a point of inflection if $\frac{\partial^{2}}{\partial x^{2}} \ln \left(h\left(x_{*}\right)\right)=0$.

\subsection{Asymptotic results}

In order to show the effect of the parameters on tails distributions, we now study the asymptotic properties of $F(x), f(x)$ and $h(x)$. First of all, let us remark that $W(y) \sim \theta y$ when $y \rightarrow 0$ and $W(y) \sim \frac{1}{(1-y)^{\theta}}$ when $y \rightarrow 1$. Therefore

$$
F(x) \sim e^{-\frac{\lambda}{\theta G(x)}}, \quad G(x) \rightarrow 0, \quad F(x) \sim e^{-\lambda[1-G(x)]^{\theta}}, \quad x \rightarrow+\infty .
$$

Similarly, we have

$$
f(x) \sim \frac{\lambda \theta g(x)}{\left(1-[1-G(x)]^{\theta}\right)^{2}} e^{-\frac{\lambda}{\theta G(x)}}, \quad G(x) \rightarrow 0
$$

and

$$
f(x) \sim \lambda \theta g(x)[1-G(x)]^{\theta-1}, \quad x \rightarrow+\infty .
$$

Concerning the hrf, the following asymptotic results hold :

$$
h(x) \sim \frac{\lambda \theta g(x)}{\left(1-[1-G(x)]^{\theta}\right)^{2}} e^{-\frac{\lambda}{\theta G(x)}}, \quad G(x) \rightarrow 0
$$

and

$$
h(x) \sim \theta \frac{g(x)}{1-G(x)}, \quad x \rightarrow+\infty .
$$

We observe that, when $x$ tends to $+\infty$, the hrf $h(x)$ is proportional to the hrf related to $G(x)$; the constant of proportionality is given by $\theta$.

\subsection{Linear representation}

Let $h>0$. We now provide useful linear representations for several functions, including $[F(x)]^{h} f(x)$. It follows from the formula of exponential power series that

$$
F(x)=\sum_{k=0}^{+\infty} \frac{1}{k !}(-1)^{k} \lambda^{k}[1-G(x)]^{\theta k}\left(1-[1-G(x)]^{\theta}\right)^{-k} .
$$


The generalized binomial series gives

$$
\left(1-[1-G(x)]^{\theta}\right)^{-k}=\sum_{\ell=0}^{+\infty}\left(\begin{array}{c}
-k \\
\ell
\end{array}\right)(-1)^{\ell}[1-G(x)]^{\theta \ell}
$$

and

$$
[1-G(x)]^{\theta(k+\ell)}=\sum_{m=0}^{+\infty}\left(\begin{array}{c}
\theta(k+\ell) \\
m
\end{array}\right)(-1)^{m}[G(x)]^{m} .
$$

Combining these equalities, we obtain the following series expansion:

$$
F(x)=\sum_{m=0}^{+\infty} a_{m} \Pi_{m}(x)
$$

where $a_{m}=(-1)^{m} \sum_{k=0}^{+\infty} \sum_{\ell=0}^{+\infty}(-1)^{k+\ell} \frac{1}{k !} \lambda^{k}\left(\begin{array}{c}-k \\ \ell\end{array}\right)\left(\begin{array}{c}\theta(k+\ell) \\ m\end{array}\right)$ and $\Pi_{m}(x)=[G(x)]^{m}$ is the cdf of the well-known Exp-G distribution with power $m$ and $\Pi_{0}(x)=1$ (see Nadarajah and Kotz (2006)). By derivation of $F(x), f(x)$ can be expressed as

$$
f(x)=\sum_{m=0}^{+\infty} a_{m+1} \pi_{m+1}(x)
$$

where $a_{m+1}=(-1)^{m+1} \sum_{k=0}^{+\infty} \sum_{\ell=0}^{+\infty}(-1)^{k+\ell} \frac{1}{k !} \lambda^{k}\left(\begin{array}{c}-k \\ \ell\end{array}\right)\left(\begin{array}{c}\theta(k+\ell) \\ m+1\end{array}\right)$ and $\pi_{m+1}(x)=(m+1)[G(x)]^{m} g(x)$ is the pdf of the Exp-G distribution with power $m+1$. Now let us observe that $[F(x)]^{h} f(x)=\frac{1}{h+1} f_{*}(x)$, where $f_{*}(x)$ denotes a pdf of the TIIGIE with parameter $(h+1) \lambda$ instead of $\lambda$. Then, by analogy with (4), we can write

$$
[F(x)]^{h} f(x)=\sum_{m=0}^{+\infty} b_{m, h} \pi_{m+1}(x)
$$

with $b_{m, h}=(-1)^{m+1} \sum_{k=0}^{+\infty} \sum_{\ell=0}^{+\infty}(-1)^{k+\ell} \frac{1}{k !} \lambda^{k}(h+1)^{k-1}\left(\begin{array}{c}-k \\ \ell\end{array}\right)\left(\begin{array}{c}\theta(k+\ell) \\ m+1\end{array}\right)$.

The series expansion (5) can be used to derive most of the mathematical properties of the TIIGIE family. This is developed in Section 4.

\section{Some special cases}

Among the numerous possible distributions arising from the TIIGIE family, we now present three special cases using classics distributions as baselines.

\subsection{TIIGIE-Uniform distribution}

We define the TIIGIE-U distribution with parameters $(\alpha, \lambda, \theta)$ the TIIGIE family with the uniform distribution on the interval $[0, \alpha]$ as baseline distribution, i.e. with 

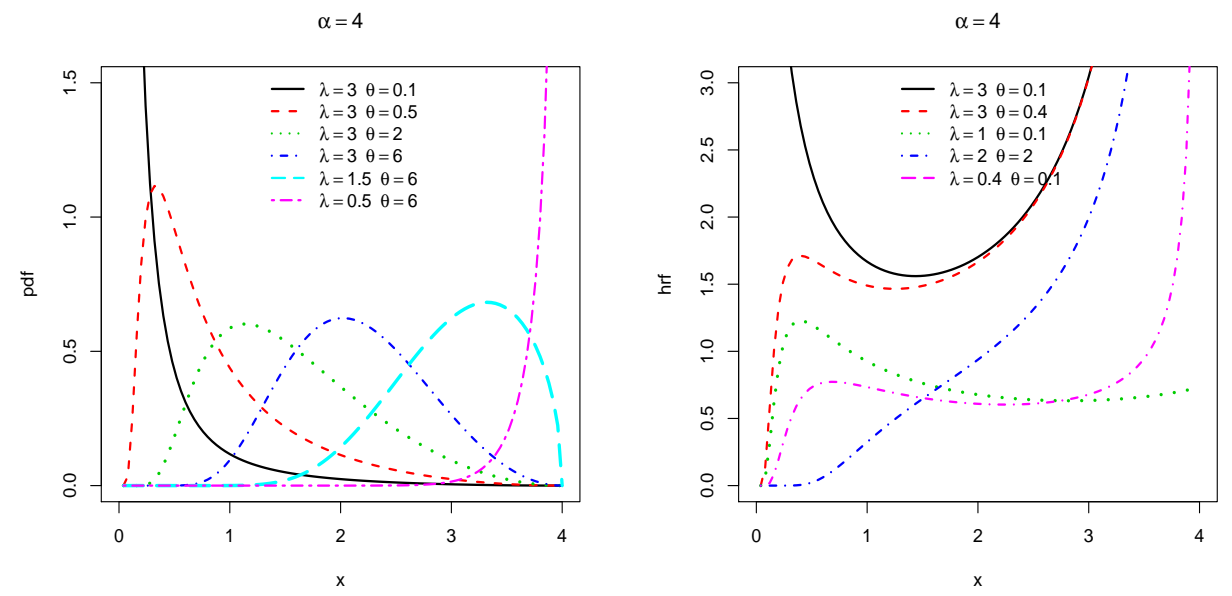

Figure 1: Plots for pdfs and hrfs of the TIIGIE-U distribution.

$\operatorname{cdf} G(x)=x / \alpha, x \in[0, \alpha], G(x)=1, x>\alpha$ and $g(x)=1 / \alpha, x \in[0, \alpha]$. The pdf of the TIIGIE-U distribution is given by

$$
f(x)=\frac{\lambda \theta[1-x / \alpha]^{\theta-1}}{\alpha\left(1-[1-x / \alpha]^{\theta}\right)^{2}} e^{-\lambda \frac{\left[1-x / \alpha \alpha^{\theta}\right.}{1-[1-x / \alpha]^{\theta}}}, \quad x \in[0, \alpha] .
$$

The associated hrf is given by

$$
h(x)=\frac{\lambda \theta[1-x / \alpha]^{\theta-1}}{\alpha\left(1-[1-x / \alpha]^{\theta}\right)^{2}\left[1-e^{-\lambda \frac{[1-x / \alpha]^{\theta}}{1-[1-\alpha / \alpha]^{\theta}}}\right]} e^{-\lambda \frac{[1-x / \alpha]^{\theta}}{1-[1-x / \alpha]^{\theta}}}, \quad x \in[0, \alpha] .
$$

Plots of the pdf and hrf of the TIIGIE-U distribution for some parameter values are displayed in Figure 1.

\subsection{TIIGIE-Weibull distribution}

We define the TIIGIE-W distribution with parameters $(\lambda, \theta, \alpha, \beta)$ the TIIGIE family with the Weibull distribution with parameters $(\alpha, \beta)$ as baseline distribution, i.e. with $\operatorname{cdf} G(x)=1-e^{-\alpha x^{\beta}}$ and $g(x)=\alpha \beta x^{\beta-1} e^{-\alpha x \beta}, x>0$. The pdf of the TIIGIE-W distribution is given by

$$
f(x)=\frac{\lambda \theta \alpha \beta x^{\beta-1} e^{-\alpha x \beta} e^{-\alpha(\theta-1) x^{\beta}}}{\left(1-e^{-\alpha \theta x^{\beta}}\right)^{2}} e^{-\lambda \frac{e^{-\alpha \theta x^{\beta}}}{1-e^{-\alpha \theta x^{\beta}}}, \quad x>0 .}
$$

The associated hrf is given by

$$
h(x)=\frac{\lambda \theta \alpha \beta x^{\beta-1} e^{-\alpha x \beta} e^{-\alpha(\theta-1) x^{\beta}}}{\left(1-e^{-\alpha \theta x^{\beta}}\right)^{2}\left[1-e^{-\lambda \frac{e^{-\alpha \theta x^{\beta}}}{1-e^{-\alpha \theta x^{\beta}}}}\right]} e^{-\lambda \frac{e^{-\alpha \theta x^{\beta}}}{1-e^{-\alpha \theta x^{\beta}}}}, \quad x>0 .
$$

Plots of the pdf and hrf of the TIIGIE-W distribution for some parameter values are displayed in Figure 2. 

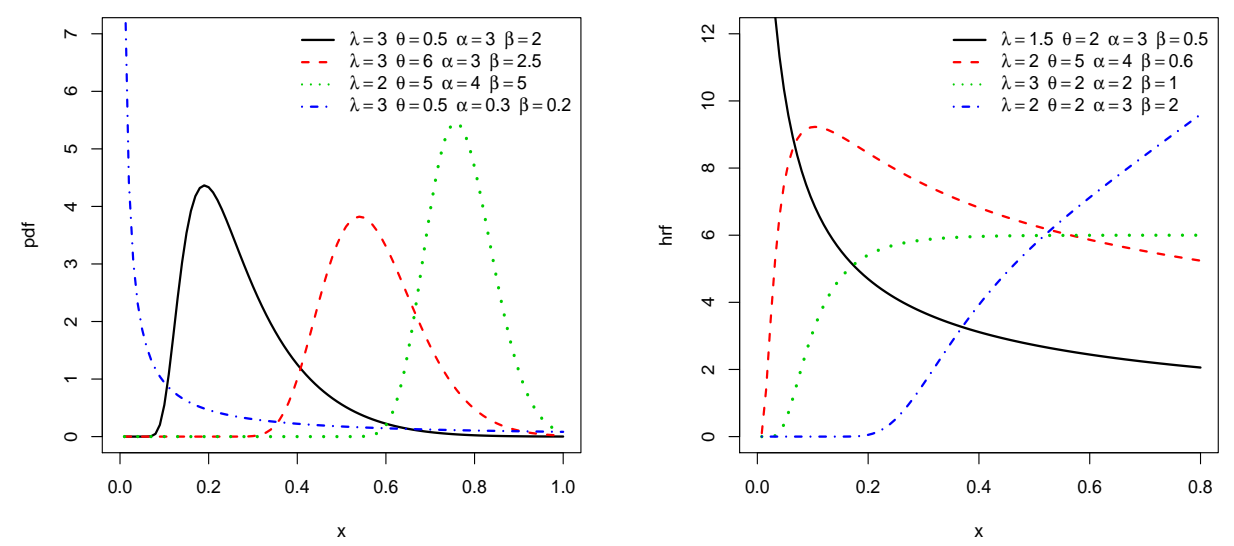

Figure 2: Plots for pdfs and hrfs of the TIIGIE-W distribution.

\subsection{TIIGIE-Burr III distribution}

We define the TIIGIE-BIII distribution with parameters $(\lambda, \theta, c, k)$ the TIIGIE family with the Burr III distribution, also known as exponentiated Lomax distribution, with parameters $(c, k)$ as baseline distribution, i.e. with $\operatorname{cdf} G(x)=\left(1+x^{-c}\right)^{-k}$ and $g(x)=c k x^{-c-1}\left(1+x^{-c}\right)^{-k-1}, x>0$. The pdf of the TIIGIE-BIII distribution is given by

$$
f(x)=\frac{\lambda \theta c k x^{-c-1}\left(1+x^{-c}\right)^{-k-1}\left[1-\left(1+x^{-c}\right)^{-k}\right]^{\theta-1}}{\left(1-\left[1-\left(1+x^{-c}\right)^{-k}\right]^{\theta}\right)^{2}} e^{-\lambda \frac{\left[1-\left(1+x^{-c}\right)^{-k}\right]^{\theta}}{1-\left[1-\left(1+x^{-c}\right)^{-k}\right]^{\theta}}}, \quad x>0 .
$$

The associated hrf is given by

$$
h(x)=\frac{\lambda \theta c k x^{-c-1}\left(1+x^{-c}\right)^{-k-1}\left[1-\left(1+x^{-c}\right)^{-k}\right]^{\theta-1}}{\left(1-\left[1-\left(1+x^{-c}\right)^{-k}\right]^{\theta}\right)^{2}\left[1-e^{-\lambda \frac{\left[1-\left(1+x^{-c)}-k\right]^{\theta}\right.}{1-\left[1-\left(1+x^{-c}\right)^{-k}\right]^{\theta}}}\right]} e^{-\lambda \frac{\left[1-\left(1+x^{-c}-k_{1}^{\theta}\right.\right.}{1-\left[1-\left(1+x^{-c}\right)^{-k}\right]^{\theta}}}, \quad x>0 .
$$

Plots of the pdf and hrf of the TIIGIE-BIII distribution for some parameter values are displayed in Figure 3. We observe shapes of different natures for the functions, which motivates us to used it as statistical model in a next section.

\section{Mathematical properties}

We now investigate some mathematical properties of the TIIGIE family. We denote by $X$ a random variable having the $\operatorname{cdf} F(x)$ (and the pdf $f(x)$ ) and by $Y$ a random variable having the $\operatorname{cdf} G(x)$ (and the pdf $g(x)$ ).

\subsection{Quantile function}

The quantile function of $X$ is given by

$$
x_{p}=G^{-1}\left[1-\left(\frac{\ln (p)}{\ln (p)-\lambda}\right)^{\frac{1}{\theta}}\right], \quad p \in(0,1] .
$$



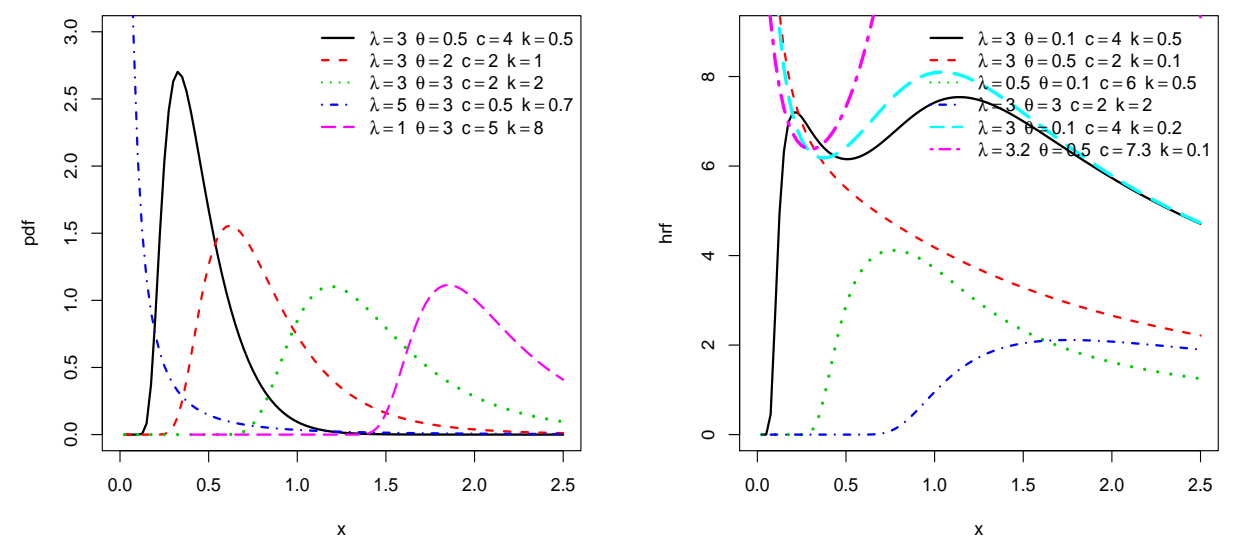

Figure 3: Plots for pdfs and hrfs of the TIIGIE-BIII distribution.

In particular, the median is given by $x_{1 / 2}=G^{-1}\left[1-\left(\frac{\ln (2)}{\ln (2)+\lambda}\right)^{\frac{1}{\theta}}\right]$. We can also use $x_{p}$ to define well-known quantile measures, as the Bowley skewness based on quartiles and the Moors kurtosis based on octiles, respectively defined by

$$
B=\frac{x_{3 / 4}+x_{1 / 4}-2 x_{1 / 2}}{x_{3 / 4}-x_{1 / 4}}, \quad M=\frac{x_{7 / 8}-x_{5 / 8}+x_{3 / 8}-x_{1 / 8}}{x_{6 / 8}-x_{2 / 8}} .
$$

Further details can be found in Kenney and Keeping (1962) and Moors (1988).

Finally, if $U$ is a random variable having the uniform distribution on the interval $(0,1)$, then the random variable $X=x_{U}$ has the cdf $F(x)$.

\subsection{Probability weighted moments}

Let $(r, h) \in(0,+\infty)^{2}$. The $(r, h)$-th probability weighted moments of $X$ and $Y$ are respectively defined by

$$
\tau_{r, h}=\mathbb{E}\left(X^{r}[F(X)]^{h}\right)=\int_{-\infty}^{+\infty} x^{r}[F(x)]^{h} f(x) d x, \quad \tau_{r, h}^{*}=\mathbb{E}\left(Y^{r}[G(Y)]^{h}\right) .
$$

Using the series expansion given by (5), we obtain

$$
\tau_{r, h}=\sum_{m=0}^{+\infty} b_{m, h} \int_{-\infty}^{+\infty} x^{r} \pi_{m+1}(x) d x=\sum_{m=0}^{+\infty} c_{m, h} \tau_{r, m}^{*}
$$

where $c_{m, h}=(m+1) b_{m, h}$. The term $\tau_{r, m}^{*}$ can be computed numerically by using the quantile function $Q_{G}(u)=G^{-1}(u)$ as $\tau_{r, m}^{*}=\int_{0}^{1}\left[Q_{G}(u)\right]^{r} u^{m} d u$. 


\subsection{Moments}

The $r$-th ordinary moment of $X$ is defined by $\mu_{r}^{\prime}=\mathbb{E}\left(X^{r}\right)=\int_{-\infty}^{+\infty} x^{r} f(x) d x$. Using (4), we can express $\mu_{r}^{\prime}$ as

$$
\mu_{r}^{\prime}=\sum_{m=0}^{+\infty} a_{m+1} \int_{-\infty}^{+\infty} x^{r} \pi_{m+1}(x) d x
$$

The integral terms can be computed numerically by noticing that $\int_{-\infty}^{+\infty} x^{r} \pi_{m+1}(x) d x=$ $(m+1) \int_{0}^{1}\left[Q_{G}(u)\right]^{r} u^{m} d u$. Alternatively, using (6), one can notice that $\mu_{r}^{\prime}=\tau_{r, 0}=$ $\sum_{m=0}^{+\infty} c_{m, 0} \tau_{r, m}^{*}$. The mean of $X$ is obtained by taking $r=1$.

Let $r$ be an integer. The $r$-th central moments of $X$ can be expressed as $\mu_{r}=$ $E\left[\left(X-\mu_{1}^{\prime}\right)^{r}\right]=\sum_{k=0}^{r}\left(\begin{array}{l}r \\ k\end{array}\right)(-1)^{k}\left(\mu_{1}^{\prime}\right)^{k} \mu_{r-k}^{\prime}$ and the $r$-th cumulants of $X$ can be obtained via the equation : $\kappa_{r}=\mu_{r}^{\prime}-\sum_{k=1}^{r-1}\left(\begin{array}{l}r-1 \\ k-1\end{array}\right) \kappa_{k} \mu_{r-k}^{\prime}$ with $\kappa_{1}=\mu_{1}^{\prime}$. The skewness of $X$ is given by $\gamma_{1}=\kappa_{3} / \kappa_{2}^{3 / 2}$ and the kurtosis of $X$ is given by $\gamma_{2}=\kappa_{4} / \kappa_{2}^{2}$.

\subsection{Moment generating function}

The moment generating function of $X$ is given by $M_{X}(t)=\mathbb{E}\left(e^{t X}\right)=\int_{-\infty}^{+\infty} e^{t x} f(x) d x$. Using (4), we can express $M_{X}(t)$ as

$$
M_{X}(t)=\sum_{m=0}^{+\infty} a_{m+1} \int_{-\infty}^{+\infty} e^{t x} \pi_{m+1}(x) d x .
$$

The integral terms can be computed numerically by noticing that $\int_{-\infty}^{+\infty} e^{t x} \pi_{m+1}(x) d x=$ $(m+1) \int_{0}^{1} e^{t Q_{G}(u)} u^{m} d u$. An alternative expression using (6) is given by

$$
M_{X}(t)=\mathbb{E}\left(e^{t X}\right)=\sum_{r=0}^{+\infty} \frac{t^{r}}{r !} \mu_{r}^{\prime}=\sum_{r=0}^{+\infty} \sum_{m=0}^{+\infty} \frac{t^{r}}{r !} c_{m, 0} \tau_{r, m}^{*} .
$$

\subsection{Incomplete moments}

The $r$-th incomplete moment of $X$ is defined by $m_{r}(t)=\mathbb{E}\left(X^{r} 1_{\{X \leq t\}}\right)=\int_{-\infty}^{t} x^{r} f(x) d x$. Using (4), we can express $m_{r}(t)$ as

$$
m_{r}(t)=\sum_{m=0}^{+\infty} a_{m+1} \int_{-\infty}^{t} x^{r} \pi_{m+1}(x) d x
$$

Again, the integral terms can be expressed as $\int_{-\infty}^{t} x^{r} \pi_{m+1}(x) d x=(m+1) \int_{0}^{G(t)}\left[Q_{G}(u)\right]^{r} u^{m} d u$. The incomplete moments of $X$ are useful tools in the definitions of several important quantities. Among them, let us mention the Lorenz curve, the Bonferroni curve, the mean deviation about the mean, the mean deviation about the median, the residual life function and the reversed residual life function. 


\subsection{Entropies}

The Rényi entropy of $X$ is defined by

$$
I_{\delta}(X)=\frac{1}{1-\delta} \log \left[\int_{-\infty}^{+\infty}[f(x)]^{\delta} d x\right],
$$

with $\delta>0$ and $\delta \neq 1$. It follows from (3) that

$$
[f(x)]^{\delta}=\frac{\lambda^{\delta} \theta^{\delta}[g(x)]^{\delta}[1-G(x)]^{(\theta-1) \delta}}{\left(1-[1-G(x)]^{\theta}\right)^{2 \delta}} e^{-\lambda \delta \frac{[1-G(x))^{\theta}}{1-[1-G(x)]^{\theta}}} .
$$

Using the exponential series expansion, we can write

$$
e^{-\lambda \delta \frac{[1-G(x)]^{\theta}}{1-[1-G(x)]^{\theta}}}=\sum_{k=0}^{+\infty} \frac{1}{k !}(-1)^{k} \lambda^{k} \delta^{k}[1-G(x)]^{\theta k}\left(1-[1-G(x)]^{\theta}\right)^{-k} .
$$

Therefore

$$
[f(x)]^{\delta}=\lambda^{\delta} \theta^{\delta}[g(x)]^{\delta} \sum_{k=0}^{+\infty} \frac{1}{k !}(-1)^{k} \lambda^{k} \delta^{k}[1-G(x)]^{\theta(k+\delta)-\delta}\left(1-[1-G(x)]^{\theta}\right)^{-(k+2 \delta)} .
$$

The generalized binomial series gives

$$
\left(1-[1-G(x)]^{\theta}\right)^{-(k+2 \delta)}=\sum_{\ell=0}^{+\infty}\left(\begin{array}{c}
-(k+2 \delta) \\
\ell
\end{array}\right)(-1)^{\ell}[1-G(x)]^{\theta \ell}
$$

and

$$
[1-G(x)]^{\theta(k+\ell+\delta)-\delta}=\sum_{m=0}^{+\infty}\left(\begin{array}{c}
\theta(k+\ell+\delta)-\delta \\
m
\end{array}\right)(-1)^{m}[G(x)]^{m} .
$$

Combining these equalities, we obtain the following series expansion:

$$
[f(x)]^{\delta}=\lambda^{\delta} \theta^{\delta} \sum_{m=0}^{+\infty} d_{m, \delta}[G(x)]^{m}[g(x)]^{\delta},
$$

where $d_{m, \delta}=(-1)^{m} \sum_{k=0}^{+\infty} \sum_{\ell=0}^{+\infty}(-1)^{k+\ell} \frac{1}{k !} \lambda^{k} \delta^{k}\left(\begin{array}{c}-(k+2 \delta) \\ \ell\end{array}\right)\left(\begin{array}{c}\theta(k+\ell+\delta)-\delta \\ m\end{array}\right)$. Therefore the Rényi entropy of TIIGIE family is given by

$$
I_{\delta}(X)=\frac{1}{1-\delta}\left[\delta \log (\lambda)+\delta \log (\theta)+\log \left[\sum_{m=0}^{+\infty} d_{m, \delta} \int_{-\infty}^{+\infty}[G(x)]^{m}[g(x)]^{\delta} d x\right]\right] .
$$

The integrals terms can be evaluated numerically for a given $G(x)$.

The $\delta$-entropy is defined by

$$
H_{\delta}(X)=\frac{1}{\delta-1} \log \left[1-\int_{-\infty}^{+\infty}[f(x)]^{\delta} d x\right],
$$

with $\delta>0$ and $\delta \neq 1$. So we have

$$
H_{\delta}(X)=\frac{1}{\delta-1} \log \left[1-\lambda^{\delta} \theta^{\delta} \sum_{m=0}^{+\infty} d_{m, \delta} \int_{-\infty}^{+\infty}[G(x)]^{m}[g(x)]^{\delta} d x\right] .
$$

Finally, the Shannon entropy of $X$ is defined by $S(X)=\mathbb{E}(-\log [f(X)])$. It is in fact a particular case of the Rényi entropy when $\delta$ tends to $1^{+}$. 


\subsection{Order statistics}

Order statistics are fundamental in many areas of statistical theory and practice. Let $X_{1}, \ldots, X_{n}$ be a random sample from the TIIGIE family. The pdf of the $i$ th order statistic, say $X_{i: n}$, is given by

$$
f_{i: n}(x)=\frac{n !}{(i-1) !(n-i) !} \sum_{j=0}^{n-i}(-1)^{j}\left(\begin{array}{c}
n-i \\
j
\end{array}\right)[F(x)]^{j+i-1} f(x) .
$$

Using the series expansion (5), we have

$$
f_{i: n}(x)=\frac{n !}{(i-1) !(n-i) !} \sum_{j=0}^{n-i}(-1)^{j}\left(\begin{array}{c}
n-i \\
j
\end{array}\right) \sum_{m=0}^{+\infty} b_{m, j+i-1} \pi_{m+1}(x) .
$$

The $r$-th ordinary moment of $X_{i: n}$ is defined by $\mu_{i: n, r}^{\prime}=\mathbb{E}\left(X_{i: n}^{r}\right)$. It follows from the above series expansion, the definition of the probability weighted moments of $Y$ and (6) that

$$
\begin{aligned}
\mu_{i: n, r}^{\prime} & =\frac{n !}{(i-1) !(n-i) !} \sum_{j=0}^{n-i}(-1)^{j}\left(\begin{array}{c}
n-i \\
j
\end{array}\right) \tau_{r, j+i-1} \\
& =\frac{n !}{(i-1) !(n-i) !} \sum_{j=0}^{n-i}(-1)^{j}\left(\begin{array}{c}
n-i \\
j
\end{array}\right) \sum_{m=0}^{+\infty} c_{m, j+i-1} \tau_{r, m}^{*}
\end{aligned}
$$

(with $\left.c_{m, j+i-1}=(m+1) b_{m, j+i-1}\right)$.

\section{Estimation inference}

\subsection{Maximum likelihood estimation (MLE)}

Let $x_{1}, \ldots, x_{n}$ be $n$ observed values from the TIIGIE family and $\xi=\left(\lambda, \theta, \xi_{*}\right)$ be the vector of unknown parameters, $\xi_{*}$ denoting the vector of parameters related to the distribution characterized by the cdf $G(x)$. The log likelihood function is given by

$$
\begin{aligned}
\ell(\xi) & =n \log (\lambda)+n \log (\theta)+\sum_{i=1}^{n} \log \left(g\left(x_{i}\right)\right)+(\theta-1) \sum_{i=1}^{n} \log \left(1-G\left(x_{i}\right)\right) \\
& -2 \sum_{i=1}^{n} \log \left(1-\left[1-G\left(x_{i}\right)\right]^{\theta}\right)-\lambda \sum_{i=1}^{n} \frac{\left[1-G\left(x_{i}\right)\right]^{\theta}}{1-\left[1-G\left(x_{i}\right)\right]^{\theta}} .
\end{aligned}
$$

The maximum likelihood estimators of the parameters are obtained by maximizing the log likelihood function. They can be obtained by solving the non-linear equations : $\frac{\partial}{\partial \lambda} \ell(\xi)=0, \frac{\partial}{\partial \theta} \ell(\xi)=0$ and $\frac{\partial}{\partial \xi_{*}} \ell(\xi)=0$ with

$$
\frac{\partial}{\partial \lambda} \ell(\xi)=\frac{n}{\lambda}-\sum_{i=1}^{n} \frac{\left[1-G\left(x_{i}\right)\right]^{\theta}}{1-\left[1-G\left(x_{i}\right)\right]^{\theta}}
$$




$$
\begin{aligned}
\frac{\partial}{\partial \theta} \ell(\xi) & =\frac{n}{\theta}+\sum_{i=1}^{n} \log \left(1-G\left(x_{i}\right)\right)+2 \sum_{i=1}^{n} \frac{\left[1-G\left(x_{i}\right)\right]^{\theta} \log \left(1-G\left(x_{i}\right)\right)}{1-\left[1-G\left(x_{i}\right)\right]^{\theta}} \\
& -\lambda \sum_{i=1}^{n} \frac{\left[1-G\left(x_{i}\right)\right]^{\theta} \log \left(1-G\left(x_{i}\right)\right)}{\left(1-\left[1-G\left(x_{i}\right)\right]^{\theta}\right)^{2}} .
\end{aligned}
$$

Let us set $g_{\xi_{*}}\left(x_{i}\right)=\frac{\partial}{\partial \xi_{*}} g\left(x_{i}\right)$ and $G_{\xi_{*}}\left(x_{i}\right)=\frac{\partial}{\partial \xi_{*}} G\left(x_{i}\right)$. Then we have

$$
\begin{aligned}
\frac{\partial}{\partial \xi_{*}} \ell(\xi) & =\sum_{i=1}^{n} \frac{g_{\xi_{*}}\left(x_{i}\right)}{g\left(x_{i}\right)}-(\theta-1) \sum_{i=1}^{n} \frac{G_{\xi_{*}}\left(x_{i}\right)}{1-G\left(x_{i}\right)} \\
& -2 \theta \sum_{i=1}^{n} \frac{\left[1-G\left(x_{i}\right)\right]^{\theta-1} G_{\xi_{*}}\left(x_{i}\right)}{1-\left[1-G\left(x_{i}\right)\right]^{\theta}}+\lambda \theta \sum_{i=1}^{n} \frac{\left[1-G\left(x_{i}\right)\right]^{\theta-1} G_{\xi_{*}}\left(x_{i}\right)}{\left(1-\left[1-G\left(x_{i}\right)\right]^{\theta}\right)^{2}}
\end{aligned}
$$

They can be solved using the Newton method or fixed point iteration methods. As usual, for determining related mathematical quantities as the variance co-variance matrix and the confidence interval for parameters, we need the information matrix which can be generated by the taking the expectation of the second order derivative.

\subsection{Least square method (LSE)}

Adopting the notations above, let $x_{(1)}, \ldots, x_{(n)}$ be $x_{1}, \ldots, x_{n}$ in increasing order. Least square estimates are obtained by minimizing the following function

$$
S(\xi)=\sum_{i=1}^{n}\left[F\left(x_{(i)}\right)-\frac{i}{n+1}\right]^{2}=\sum_{i=1}^{n}\left[e^{-\lambda \frac{\left[1-G\left(x_{(i)}\right)\right]^{\theta}}{1-\left[1-G\left(x_{(i)}\right)\right]^{\theta}}}-\frac{i}{n+1}\right]^{2} .
$$

Minimizing $S(\Theta)$ with respect to $\lambda, \theta$ and $\xi$, we have following system of non linear equations:

$$
\begin{aligned}
& \frac{\partial S(\xi)}{\partial \lambda}=-2 \sum_{i=1}^{n}\left[e^{-\lambda \frac{\left[1-G\left(x_{(i)}\right)\right]^{\theta}}{1-\left[1-G\left(x_{(i)}\right)\right]^{\theta}}}-\frac{i}{n+1}\right] \frac{\left[1-G\left(x_{(i)}\right)\right]^{\theta}}{1-\left[1-G\left(x_{(i)}\right)\right]^{\theta}} e^{-\lambda \frac{\left[1-G\left(x_{i}\right)\right]^{\theta}}{1-\left[1-G\left(x_{(i)}\right)\right]^{\theta}}}=0, \\
& \frac{\partial S(\xi)}{\partial \theta}=-2 \lambda \sum_{i=1}^{n}\left[e^{-\lambda \frac{\left[1-G\left(x_{(i)}\right)\right]^{\theta}}{1-\left[1-G\left(x_{(i)}\right)\right]^{\theta}}}-\frac{i}{n+1}\right] \frac{\left[1-G\left(x_{(i)}\right)\right]^{\theta} \log \left(1-G\left(x_{(i)}\right)\right)}{\left(1-\left[1-G\left(x_{(i)}\right)\right]^{\theta}\right)^{2}} e^{-\lambda \frac{\left[1-G\left(x_{(i)}\right)\right]^{\theta}}{1-[1-G(x(i))]^{\theta}}} \\
& =0 \text {, } \\
& \frac{\partial S(\xi)}{\partial \xi_{*}}=2 \lambda \theta \sum_{i=1}^{n}\left[e^{-\lambda \frac{\left[1-G\left(x_{(i)}\right)\right]^{\theta}}{1-\left[1-G\left(x_{(i)}\right)\right]^{\theta}}}-\frac{i}{n+1}\right] \frac{\left[1-G\left(x_{(i)}\right)\right]^{\theta-1} G_{\xi_{*}}\left(x_{(i)}\right)}{\left(1-\left[1-G\left(x_{(i)}\right)\right]^{\theta}\right)^{2}} e^{-\lambda \frac{\left[1-G\left(x_{(i)}\right)\right]^{\theta}}{1-\left[1-G\left(x_{(i)}\right)\right]^{\theta}}}=0 .
\end{aligned}
$$

This system of non-linear equations can be solved numerically by any software to obtained the estimates. 


\subsection{A simulation study}

In order to assess the performance of the maximum likelihood and least square methods for estimating the parameters, a small simulation is carried out. For such purposes, a TIIGIE-BIII distribution is considered using Monte Carlo simulations. The process is carried out as follow:

- The number of Monte Carlo replications was made 1000 times each with sample sizes $n=30,50,100$ and 300 .

- Initial values for the parameters are selected as given in Tables 1, 2, 3 and 4,

- The formula for the mean squared error (MSE) of the estimate $\widehat{\lambda}$ of $\lambda$ is given by

$$
\frac{1}{1000} \sum_{i=1}^{1000}(\widehat{\lambda}-\lambda)^{2}
$$

- The third step is also repeated for the other parameters.

These numerical results show that the considered estimation methods perform quite well in estimating the model parameters of the TIIGIE-BIII distribution.

Table 1: Estimates and MSEs of TIIGIE-BIII distribution for ML and LS estimate, Set $1:(\lambda, \theta, c, k)=$ $(0.5,0.5,0.5,0.5)$ and Set $2:(\lambda, \theta, c, k)=(0.5,0.5,0.5,1.5)$.

\begin{tabular}{c|cc|cc||cc|cc|}
\hline & Set 1 & \multicolumn{3}{|c||}{ Set 2 } & & & \\
\hline & MLE & & LSE & & MLE & & LSE & \\
\hline$n$ & Estimates & MSEs & Estimates & MSEs & Estimates & MSEs & Estimates & MSEs \\
\hline 30 & 0.5215 & 0.0100 & 0.499743 & 0.000236 & 0.5124 & 0.0092 & 0.499918 & 0.000233 \\
& 0.5242 & 0.0224 & 0.500959 & 0.000412 & 0.5350 & 0.0355 & 0.500669 & 0.000411 \\
& 0.5415 & 0.0277 & 0.497127 & 0.000047 & 0.5275 & 0.0161 & 0.498370 & 0.000189 \\
& 0.5411 & 0.0224 & 0.498763 & 0.000255 & 1.5844 & 0.1707 & 1.496670 & 0.002238 \\
\hline 50 & 0.5148 & 0.0061 & 0.500492 & 0.000138 & 0.5064 & 0.0052 & 0.499829 & 0.000139 \\
& 0.5127 & 0.0104 & 0.499697 & 0.000241 & 0.5228 & 0.0119 & 0.500617 & 0.000246 \\
& 0.5262 & 0.0140 & 0.498323 & 0.000025 & 0.5181 & 0.0084 & 0.499235 & 0.000114 \\
& 0.5274 & 0.0130 & 0.499869 & 0.000147 & 1.5530 & 0.1020 & 1.497740 & 0.001352 \\
\hline 100 & 0.5055 & 0.0026 & 0.500618 & 0.000067 & 0.5080 & 0.0027 & 0.499670 & 0.000070 \\
& 0.5074 & 0.0045 & 0.499349 & 0.000114 & 0.5044 & 0.0043 & 0.500584 & 0.000120 \\
& 0.5122 & 0.0063 & 0.499296 & 0.000011 & 0.5050 & 0.0033 & 0.499720 & 0.000056 \\
& 0.5111 & 0.0055 & 0.500341 & 0.000071 & 1.5414 & 0.0500 & 1.497980 & 0.000680 \\
\hline 300 & 0.5024 & 0.0008 & 0.499964 & 0.000023 & 0.5001 & 0.0009 & 0.499920 & 0.000023 \\
& 0.5009 & 0.0012 & 0.500108 & 0.000039 & 0.5044 & 0.0015 & 0.500166 & 0.000039 \\
& 0.5015 & 0.0018 & 0.499727 & $3.473 \times 10^{-6}$ & 0.5016 & 0.0012 & 0.499932 & 0.000019 \\
& 0.5028 & 0.0016 & 0.499873 & 0.000024 & 1.5030 & 0.0163 & 1.499490 & 0.000218 \\
\hline
\end{tabular}


Table 2: Estimates and MSEs of TIIGIE-BIII distribution for ML and LS estimate, Set $3:(\lambda, \theta, c, k)=$ $(0.5,0.5,1.5,0.5)$ and Set $4:(\lambda, \theta, c, k)=(0.5,0.5,1.5,1.5)$.

\begin{tabular}{c|cc|cc||cc|cr|}
\hline & Set 3 & \multicolumn{3}{|c||}{ Set 4} \\
\hline & MLE & & LSE & & MLE & & \multicolumn{2}{l|}{ LSE } \\
\hline$n$ & Estimates & MSEs & Estimates & MSEs & Estimates & MSEs & Estimates & MSEs \\
\hline 30 & 0.5166 & 0.0100 & 0.499328 & 0.000216 & 0.5191 & 0.0098 & 0.499299 & 0.000226 \\
& 0.5348 & 0.0284 & 0.501433 & 0.000386 & 0.5306 & 0.0275 & 0.501402 & 0.000407 \\
& 1.6011 & 0.2175 & 1.491430 & 0.000429 & 1.5585 & 0.1327 & 1.496130 & 0.001638 \\
& 0.5328 & 0.0209 & 0.498370 & 0.000235 & 1.6076 & 0.1921 & 1.494550 & 0.002219 \\
\hline \multirow{3}{*}{50} & 0.5142 & 0.0059 & 0.499610 & 0.000132 & 0.5104 & 0.0053 & 0.498955 & 0.000141 \\
& 0.5127 & 0.0110 & 0.500805 & 0.000231 & 0.5147 & 0.0101 & 0.501748 & 0.000250 \\
& 1.5575 & 0.1177 & 1.494350 & 0.000232 & 1.5342 & 0.0698 & 1.499930 & 0.001049 \\
& 0.5231 & 0.0128 & 0.498973 & 0.000143 & 1.5615 & 0.1043 & 1.495020 & 0.001354 \\
\hline \multirow{2}{*}{100} & 0.5054 & 0.0025 & 0.499889 & 0.000068 & 0.5045 & 0.0027 & 0.499892 & 0.000068 \\
& 0.5078 & 0.0046 & 0.500354 & 0.000118 & 0.5097 & 0.0049 & 0.500294 & 0.000118 \\
& 1.5368 & 0.0539 & 1.497690 & 0.000103 & 1.5260 & 0.0321 & 1.498750 & 0.000511 \\
& 0.5114 & 0.0054 & 0.499619 & 0.000073 & 1.5319 & 0.0503 & 1.498760 & 0.000656 \\
\hline 300 & 0.5023 & 0.0009 & 0.499811 & 0.000024 & 0.5032 & 0.0009 & 0.499748 & 0.000024 \\
& 0.5017 & 0.0014 & 0.500324 & 0.000042 & 0.5008 & 0.0015 & 0.500384 & 0.000041 \\
& 1.5125 & 0.0179 & 1.499100 & 0.000036 & 1.5031 & 0.0104 & 1.500160 & 0.000176 \\
& 0.5045 & 0.0019 & 0.499715 & 0.000026 & 1.5150 & 0.0158 & 1.498920 & 0.000225 \\
\hline
\end{tabular}

Table 3: Estimates and MSEs of TIIGIE-BIII distribution for ML and LS estimate, Set $5:(\lambda, \theta, c, k)=$ $(0.5,1.5,0.5,0.5)$ and Set $6:(\lambda, \theta, c, k)=(0.5,1.5,0.5,1.5)$.

\begin{tabular}{c|cc|cc||cc|cc|}
\hline & Set 5 & \multicolumn{7}{|c||}{ Set 6} \\
\hline & MLE & & LSE & & MLE & & LSE \\
\hline$n$ & Estimates & MSEs & Estimates & MSEs & Estimates & MSEs & Estimates & MSEs \\
\hline 30 & 0.5162 & 0.0100 & 0.499505 & 0.000225 & 0.5159 & 0.0095 & 0.499355 & 0.000222 \\
& 1.6094 & 0.2849 & 1.503770 & 0.003637 & 1.5969 & 0.2164 & 1.504000 & 0.003528 \\
& 0.6297 & 0.2824 & 0.497733 & 0.000628 & 0.5453 & 0.0336 & 0.496525 & 0.000062 \\
& 0.6335 & 0.2289 & 0.498864 & 0.000837 & 1.8424 & 2.1402 & 1.495190 & 0.007483 \\
\hline 50 & 0.5125 & 0.0055 & 0.499409 & 0.000143 & 0.5108 & 0.0058 & 0.500272 & 0.000138 \\
& 1.5424 & 0.0925 & 1.503630 & 0.002285 & 1.5519 & 0.0955 & 1.500110 & 0.002137 \\
& 0.5787 & 0.0698 & 0.498205 & 0.000400 & 0.5294 & 0.0189 & 0.498372 & 0.000034 \\
& 0.5766 & 0.0642 & 0.498779 & 0.000532 & 1.7171 & 0.6178 & 1.501180 & 0.004643 \\
\hline 100 & 0.5038 & 0.0024 & 0.499805 & 0.000070 & 0.5021 & 0.0026 & 0.499853 & 0.000069 \\
& 1.5289 & 0.0410 & 1.501350 & 0.001082 & 1.5385 & 0.0472 & 1.501100 & 0.001083 \\
& 0.5297 & 0.0192 & 0.499253 & 0.000196 & 0.5108 & 0.0078 & 0.499046 & 0.000016 \\
& 0.5278 & 0.0181 & 0.499578 & 0.000262 & 1.5693 & 0.1732 & 1.498960 & 0.002344 \\
\hline 300 & 0.5016 & 0.0008 & 0.499653 & 0.000023 & 0.5018 & 0.0009 & 0.500171 & 0.000024 \\
& 1.5071 & 0.0123 & 1.501500 & 0.000365 & 1.5107 & 0.0128 & 1.499460 & 0.000367 \\
& 0.5083 & 0.0050 & 0.499227 & 0.000066 & 0.5085 & 0.0025 & 0.499678 & $5.62 \times 10^{-6}$ \\
& 0.5088 & 0.0051 & 0.499285 & 0.000088 & 1.5337 & 0.0471 & 1.500840 & 0.000811 \\
\hline
\end{tabular}


Table 4: Estimates and MSEs of TIIGIE-BIII distribution for ML and LS estimate, Set $7:(\lambda, \theta, c, k)=$ $(0.5,1.5,1.5,0.5)$ and Set $8:(\lambda, \theta, c, k)=(0.5,1.5,1.5,1.5)$.

\begin{tabular}{c|cc|cc||cc|cc|}
\hline & Set 7 & & \multicolumn{1}{|c||}{ Set 8 } & & & \\
\hline & MLE & & LSE & & MLE & & LSE & \\
\hline$n$ & Estimates & MSEs & Estimates & MSEs & Estimates & MSEs & Estimates & MSEs \\
\hline 30 & 0.5197 & 0.0097 & 0.499755 & 0.000229 & 0.5208 & 0.0103 & 0.500157 & 0.000217 \\
& 1.5799 & 0.2044 & 1.502770 & 0.003680 & 1.6034 & 0.2964 & 1.500630 & 0.003419 \\
& 1.8957 & 1.5805 & 1.494160 & 0.005612 & 1.6689 & 0.3168 & 1.489960 & 0.000540 \\
& 0.6350 & 0.1871 & 0.499309 & 0.000847 & 2.0019 & 4.9282 & 1.499610 & 0.007306 \\
\hline 50 & 0.5137 & 0.0056 & 0.499456 & 0.000136 & 0.5114 & 0.0052 & 0.500055 & 0.000142 \\
& 1.5459 & 0.1737 & 1.503070 & 0.002183 & 1.5446 & 0.0993 & 1.500940 & 0.002226 \\
& 1.7327 & 0.5926 & 1.494290 & 0.003449 & 1.5943 & 0.1666 & 1.494680 & 0.000317 \\
& 0.5781 & 0.0631 & 0.498780 & 0.000505 & 1.7021 & 0.4618 & 1.499980 & 0.004812 \\
\hline 100 & 0.5037 & 0.0027 & 0.499946 & 0.000072 & 0.5038 & 0.0028 & 0.500301 & 0.000068 \\
& 1.5292 & 0.0435 & 1.500770 & 0.001135 & 1.5313 & 0.0476 & 1.499270 & 0.001043 \\
& 1.5764 & 0.1836 & 1.498230 & 0.001807 & 1.5378 & 0.0762 & 1.497370 & 0.000143 \\
& 0.5266 & 0.0214 & 0.499813 & 0.000270 & 1.5874 & 0.2076 & 1.501440 & 0.002286 \\
\hline 300 & 0.5010 & 0.0008 & 0.499997 & 0.000021 & 0.5022 & 0.0008 & 0.500050 & 0.000022 \\
& 1.5111 & 0.0127 & 1.500170 & 0.000328 & 1.5039 & 0.0111 & 1.499910 & 0.000344 \\
& 1.5270 & 0.0486 & 1.499550 & 0.000540 & 1.5148 & 0.0214 & 1.498900 & 0.000045 \\
& 0.5088 & 0.0054 & 0.499972 & 0.000080 & 1.5310 & 0.0420 & 1.500120 & 0.000747 \\
\hline
\end{tabular}




\section{Applications}

In this section, we prove empirically the flexibility of the TIIGIE-BIII distributions by means of three real data sets. The TIIGIE-BIII distribution will be compared with some competitive models listed in Table 5. The pdfs of these models are given in Appendix. We consider the $-\widehat{\ell}$ (where $\widehat{\ell}$ the maximized log-likelihood), AIC (Akaike information criterion), BIC (Bayesian information criterion), CVM (Cramér-Von Mises), $\mathrm{AD}$ (Anderson-Darling) and KS (Kolmogorov Smirnov with its p-value (PV)) statistics to compare the fitted distributions. The results in this section are obtained using the R PROGRAM.

Table 5: The competitive models of the TIIGIE-BIII distribution.

\begin{tabular}{ll}
\hline \hline Distribution & Author(s) \\
\hline \hline Type II General Exponential-Lomax (TIIGE-Lx) & Hamedani et al. $(2018)$ \\
Odd Frèchet-Lomax (OFr-Lx) & Haq and Elgarhy (2018) \\
Weubull Lomax (WLx) & Tahir et aL (2015) \\
Kumaraswamy Lomax (KwLx) & Lemonte and Cordeiro (2013) \\
Beta Lomax (BLx) & Lemonte and Cordeiro (2013) \\
Exponentiated Lomax (ELx) & Abdul-Moniem and Abdel-Hameed (2012) \\
\hline \hline
\end{tabular}

Data set 1 : Mead (2016) used actual taxes data set. The data consists of the monthly actual taxes revenue in Egypt from January 2006 to November 2010. The distribution is highly skewed to the right. The actual taxes revenue data (in 1000 million Egyptian pounds) are: 5.9, 20.4, 14.9, 16.2, 17.2, 7.8, 6.1, 9.2, 10.2, 9.6, 13.3, 8.5, 21.6, $18.5,5.1,6.7,17,8.6,9.7,39.2,35.7,15.7,9.7,10,4.1,36,8.5,8,9.2,26.2,21.9,16.7$, $21.3,35.4,14.3,8.5,10.6,19.1,20.5,7.1,7.7,18.1,16.5,11.9,7,8.6,12.5,10.3,11.2$, $6.1,8.4,11,11.6,11.9,5.2,6.8,8.9,7.1,10.8$.

Data set 2 : The data contain Failure stresses of bundles of 1000 impregnated carbon fibers Length $20 \mathrm{~mm}$ from Crowder et al. (1991).

2.526, 2.546, 2.628, 2.628, 2.669, 2.669, 2.71, 2.731, 2.731, 2.731, 2.752, 2.752, 2.793, $2.834,2.834,2.854,2.875,2.875,2.895,2.916,2.916,2.957,2.977,2.998,3.06,3.06$, 3.06, 3.08.

Table 6: Goodness-of-fit measures for data set 1.

\begin{tabular}{lccccccc}
\hline \hline Model & $-\widehat{\ell}$ & $\mathrm{AIC}$ & $\mathrm{BIC}$ & $\mathrm{CVM}$ & $\mathrm{AD}$ & $\mathrm{KS}$ & $\mathrm{PV}$ \\
\hline TIIGIE-BIII & 187.0751 & 383.1501 & 391.4603 & 0.0370 & 0.2396 & 0.0564 & 0.9919 \\
TIIGE-Lx & 198.7821 & 405.5642 & 413.8743 & 0.3228 & 2.0627 & 0.1419 & 0.1857 \\
OFr-Lx & 190.0885 & 385.1771 & 398.4097 & 0.0401 & 0.2487 & 0.0609 & 0.9809 \\
WLx & 193.9537 & 395.9074 & 404.2175 & 0.2105 & 1.3182 & 0.1282 & 0.2866 \\
KwLx & 187.9425 & 383.8849 & 392.1951 & 0.0413 & 0.2641 & 0.0658 & 0.9800 \\
BLx & 188.3614 & 384.7228 & 393.0329 & 0.0436 & 0.2627 & 0.0628 & 0.9738 \\
ELx & 189.9118 & 384.8235 & 391.9961 & 0.0422 & 0.2575 & 0.0674 & 0.9510 \\
\hline \hline
\end{tabular}


Table 7: MLEs and SEs (in parentheses) for data set 1.

\begin{tabular}{lcccc}
\hline \hline Model & \multicolumn{4}{c}{ Estimates } \\
\hline TIIGIE-BIII & 1.3035 & 16.0723 & 0.5361 & 11.3254 \\
$(\lambda, \theta, c, k)$ & $(0.3901)$ & $(2.8563)$ & $(0.2819)$ & $(0.0610)$ \\
TIIGE-Lx & 0.1892 & 0.0083 & 1.4577 & 10.3473 \\
$(\lambda, \alpha, a, b)$ & $(0.0155)$ & $(0.0032)$ & $(0.0128)$ & $(0.0225)$ \\
OFr-Lx & 1.6698 & 66.9329 & 5.2782 & \\
$(\theta, \alpha, \beta)$ & $(0.2798)$ & $(9.3477)$ & $(0.1088)$ & \\
WLx & 0.1782 & 1.9231 & 17.3346 & 3.7849 \\
$(\alpha, \beta, a, b)$ & $(0.0916)$ & $(2.7434)$ & $(3.2247)$ & $(1.4321)$ \\
KwLx & 83.5491 & 129.4375 & 51.6285 & 0.2297 \\
$(\alpha, \beta, a, b)$ & $(2.8877)$ & $(3.3168)$ & $(16.1895)$ & $(0.0347)$ \\
BLx & 9.6404 & 5.6777 & 29.3087 & 0.6645 \\
$(\alpha, \beta, a, b)$ & $(0.9320)$ & $(2.6266)$ & $(0.3286)$ & $(0.4529)$ \\
ELx & 3.2130 & 4.2546 & 41.9873 & \\
$(\alpha, \beta, a)$ & $(1.4229)$ & $(6.5601)$ & $(0.6488)$ & \\
\hline \hline
\end{tabular}

Tables 6 and 8 provide the values of goodness-of-fit measures for the TIIGIE-BIII model and other fitted models, whereas the MLEs and their corresponding standard errors (SEs) (in parentheses) are listed in Tables 7 and 9, respectively. The plots of the fitted model is shown in Figures 4 and 5. These plots and Tables 6 and 8 indicate that the TIIGIE-BIII model yields the best fit to the others competitive models.

Table 8: Goodness-of-fit measures for data set 2.

\begin{tabular}{lccccccc}
\hline \hline Model & $-\widehat{\ell}$ & AIC & BIC & CVM & AD & KS & PV \\
\hline TIIGIE-BIII & -12.5338 & -16.8677 & -11.9389 & 0.0333 & 0.2513 & 0.0911 & 0.9642 \\
TIIGE-Lx & -10.5754 & -13.1508 & -7.8219 & 0.0516 & 0.3876 & 0.1310 & 0.7226 \\
OFr-Lx & -10.6272 & -15.2544 & -11.2577 & 0.0650 & 0.4817 & 0.1189 & 0.8231 \\
WLx & -11.8302 & -15.6604 & -10.3316 & 0.0597 & 0.4468 & 0.1281 & 0.7478 \\
KwLx & -12.0054 & -16.0809 & -11.3521 & 0.0408 & 0.3142 & 0.1241 & 0.7816 \\
BLx & -12.4135 & -16.8270 & -11.4982 & 0.0398 & 0.2633 & 0.0999 & 0.9426 \\
ELx & -10.9943 & -15.9886 & -11.9920 & 0.0575 & 0.4291 & 0.1144 & 0.8567 \\
\hline \hline
\end{tabular}


Table 9: MLEs and SEs (in parentheses) for data set 2.

\begin{tabular}{lcccc}
\hline \hline Model & \multicolumn{4}{c}{ Estimates } \\
\hline TIIGIE-BIII & 2.2471 & 15.0705 & 1.6547 & 41.7735 \\
$(\lambda, \theta, c, k)$ & $(0.0852)$ & $(0.5356)$ & $(0.8421)$ & $(6.7835)$ \\
TIIGE-Lx & 40.0767 & 40.0138 & 25.0314 & 15.1712 \\
$(\lambda, \alpha, a, b)$ & $(0.4884)$ & $(0.4900)$ & $(5.1094)$ & $(0.0391)$ \\
OFr-Lx & 14.2219 & 27.2773 & 7.2345 & \\
$(\theta, \alpha, \beta)$ & $(3.2679)$ & $(0.8324)$ & $(2.02942)$ & \\
WLx & 0.8702 & 2.7291 & 15.8424 & 20.9831 \\
$(\alpha, \beta, a, b)$ & $(0.1116)$ & $(1.4434)$ & $(0.0287)$ & $(1.9301)$ \\
KwLx & 92.56841 & 67.6157 & 145.5810 & 77.3110 \\
$(\alpha, \beta, a, b)$ & $(1.3827)$ & $(2.9101)$ & $(1.9805)$ & $(5.0347)$ \\
BLx & 87.7305 & 39.5196 & 8.0644 & 155.9390 \\
$(\alpha, \beta, a, b)$ & $(0.4855)$ & $(1.9286)$ & $(1.8289)$ & $(2.1959)$ \\
ELx & 288.9114 & 129.9128 & 145.7873 & \\
$(\alpha, \beta, a)$ & $(12.9229)$ & $(8.5911)$ & $(6.9498)$ & \\
\hline \hline
\end{tabular}
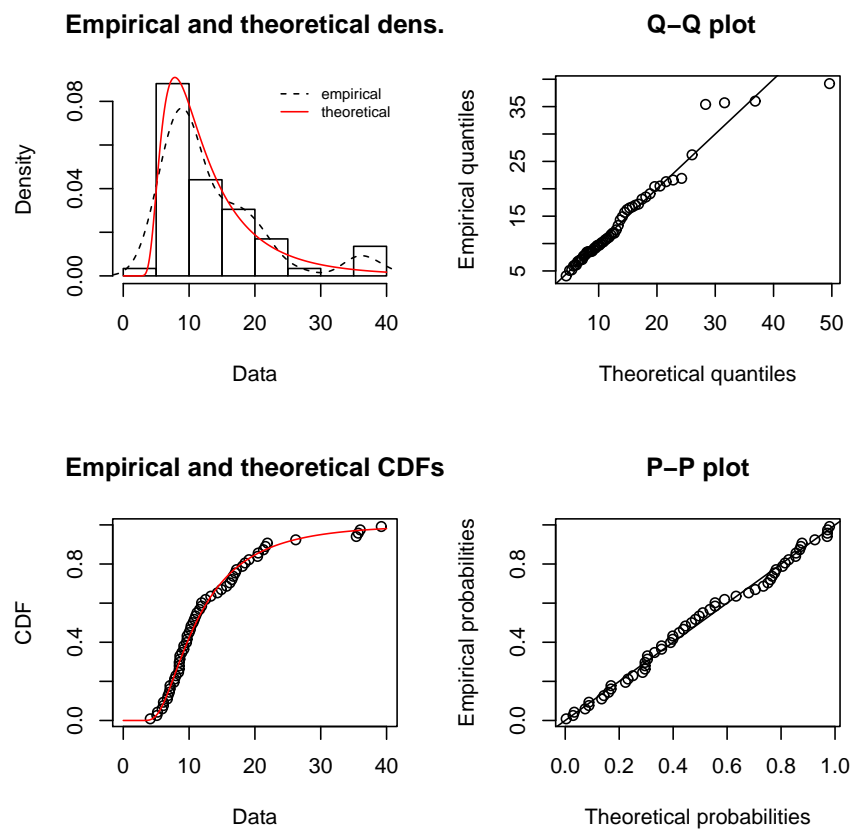

Figure 4: PP, QQ, epdf and ecdf plots of the TIIGIE-BIII distribution for data set 1. 

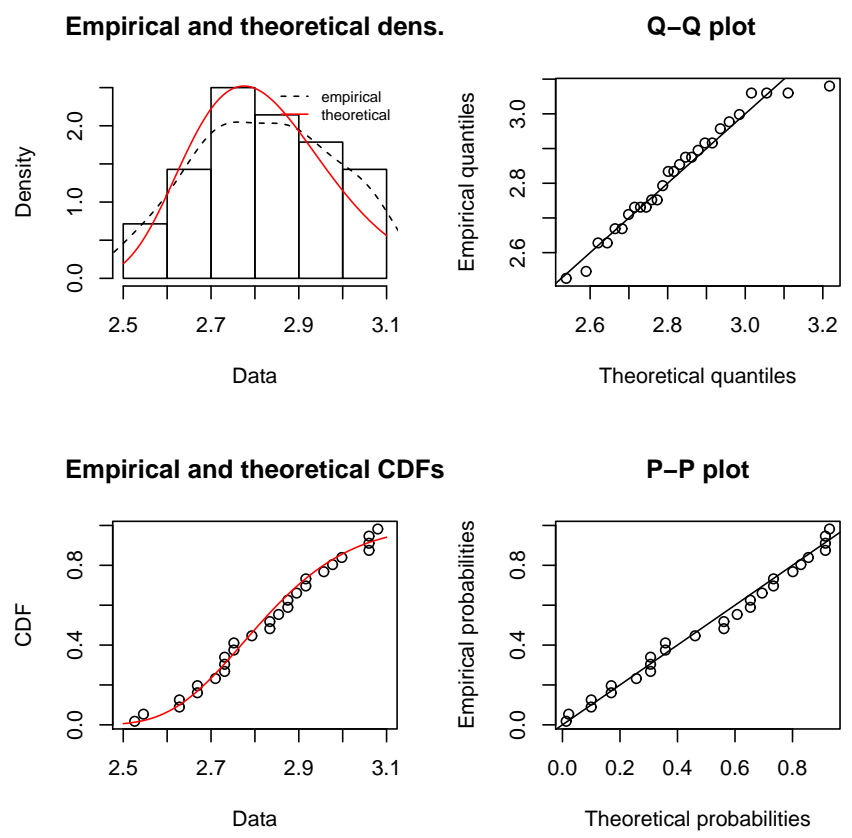

Figure 5: PP, QQ, epdf and ecdf plots of the TIIGIE-BIII distribution for data set 2. 


\section{Appendix}

The pdfs of the statistical models used in Section 6 are presented below.

- The pdf of the ELx distribution introduced by Abdul-Moniem and Abdel-Hameed (2012) is given by

$$
f(x)=\frac{a \alpha}{\beta}\left[1+\frac{x}{\beta}\right]^{-(\alpha+1)}\left\{1-\left[1+\frac{x}{\beta}\right]^{-\alpha}\right\}^{a-1}, \quad x>0 .
$$

- The pdf of the BLx distribution introduced by Lemonte and Cordeiro (2013) is given by

$$
f(x)=\frac{\alpha}{\beta B(a, b)}\left[1+\frac{x}{\beta}\right]^{-(\alpha b+1)}\left\{1-\left[1+\frac{x}{\beta}\right]^{-\alpha}\right\}^{a-1}, \quad x>0,
$$

where $B(a, b)=\int_{0}^{1} x^{a-1}(1-x)^{b-1} d x$.

- The pdf of the KwLx distribution introduced by Lemonte and Cordeiro (2013) is given by

$$
\begin{aligned}
f(x)= & \frac{a b \alpha}{\beta}\left[1+\frac{x}{\beta}\right]^{-(\alpha+1)}\left\{1-\left[1+\frac{x}{\beta}\right]^{-\alpha}\right\}^{a-1} \times \\
& {\left[1-\left\{1-\left[1+\frac{x}{\beta}\right]^{-\alpha}\right\}^{a}\right]^{b-1}, \quad x>0 . }
\end{aligned}
$$

- The pdf of the TIIGE-Lx distribution introduced by Hamedani et al. (2018) is given by

$$
f(x)=\lambda \alpha \frac{a}{b}\left(1+\frac{x}{b}\right)^{-(a+1)}\left(1+\frac{x}{b}\right)^{a(\alpha+1)} e^{\lambda\left\{1-\left(1+\frac{x}{b}\right)^{a \alpha}\right\}}, \quad x>0 .
$$

- The pdf of the OFr-Lx distribution introduced by Haq and Elgarhy (2018) is given by

$$
f(x)=\frac{\alpha \theta[1+(x / \beta)]^{-(\alpha \theta+1)}}{\beta\left[1-[1+(x / \beta)]^{-\alpha}\right]^{\theta+1}} e^{-\left\{\frac{[1+(x / \beta)]^{-\alpha}}{1-[1+(x / \beta)]^{-\alpha}}\right\}^{\theta}}, \quad x>0 .
$$

- The pdf of the WLx distribution introduced by Tahir et aL (2015) is given by

$$
f(x)=\frac{a b \alpha}{\beta}\left[1+\frac{x}{\beta}\right]^{b \alpha-1}\left\{1-\left[1+\frac{x}{\beta}\right]^{-\alpha}\right\}^{b-1} e^{-a\left\{\left[1+\frac{x}{\beta}\right]^{\alpha}-1\right\}^{b}}, \quad x>0 .
$$




\section{References}

Abdul-Moniem, I.B. and Abdel-Hameed, H.F. (2012). On exponentiated Lomax distribution, International Journal of Mathematical Archive 3, 2144-2150.

Alzaatreh, A., Famoye, F. and Lee, C. (2013). A new method for generating families of continuous distributions. Metron, 71, 63-79.

Alzaatreh, A., Famoye, F. and Lee, C. (2014). The gamma-normal distribution: Properties and applications. Computational Statistics and Data Analysis, 69, 67-80.

Alzaghal, A., Lee, C. and Famoye, F. (2013). Exponentiated T-X family of distributions with some applications. International Journal of Probability and Statistics, 2, 31-49.

Bourguignon, M., Silva, R.B. and Cordeiro, G.M. (2014). The Weibull-G family of probability distributions. Journal of Data Science, 12, 53-68.

Cordeiro, G.M., Ortega, E.M.M. and da Cunha, D.C.C. (2013). The exponentiated generalized class of distributions. Journal of Data Science, 11, 1-27.

Crowder, M.J., Kimber, A.C., Smith, R.L. and Sweeting, T.J. (1991). Statistical Analysis of Reliability Data. Chapman and Hall, UK.

Gupta, R.C., Gupta, P. L., and Gupta, R.D. (1998). Modeling failure time data by Lehman alternatives. Communications in Statistics-Theory and methods, 27(4), 887904.

Hamedani, G.G., Rasekhi, M., Najibi, S.M., Yousof, H.M and Alizadeh, M. (2018). Type II General Exponential Class of Distributions, Accepted in PJSOR.

Haq, M.A.U. and Elgarhy, M. (2018). The Odd Frêchet-G Family of Probability Distributions. J. Stat. Appl., 7, 1, 185-201.

Kenney, J.F. and Keeping, E.S. (1962). Mathematics of Statistics, 3 edn, Chapman and Hall Ltd, New Jersey.

Lemonte, A.J. and Cordeiro, G.M. (2013). An extended Lomax distribution, Statistics, $47,4,800-816$.

Mead, M.E. (2016). On five- parameter Lomax distribution: properties and applications, Pakistan Journal of Statistics and Operations Research, 1, 185-199.

Moors, J.J. (1988). A quantile alternative for kurtosis. Journal of the Royal Statistical Society: Series D, 37, 25-32.

Nadarajah, S. and Kotz, S. (2006). The Exponentiated-type Distributions. Acta Applicandae Mathematicae, 92, 97-111.

Nasir, A., Aljarrah, M., Jamal, F. and Tahir, M.H. (2017). A New Generalized Burr Family of Distributions Based on Quantile Function. J. Stat. Appl. Pro., 6, 3, 499504. 
Tahir, M.H., Cordeiro, G.M., Mansoor, M and Zubair, M. (2015).The Weibull-Lomax distribution: properties and applications, Hacettepe Journal of Mathematics and Statistics, 44, 2, 461-480.

Torabi, H. and Montazari, N.H. (2014). The logistic-uniform distribution and its application. Communications in Statistics-Simulation and Computation, 43, 2551-2569. 